\title{
WRAPPINGS OF PERMUTATIONS
}

\author{
SAUL STAHL
}

\begin{abstract}
A theory of wrappings of permutations is constructed which is analogous to the well-known concept of branched coverings of Riemann surfaces. It is shown that this theory is strong enough to contain combinatorial definitions of such well-known groups as Fuchsian groups of the first kind and triangle groups.
\end{abstract}

Introduction. The proofs of several classical theorems of mathematics employ the device of embedding a combinatorial graph in the plane and then making use of its discrete structure. The proof of the Jordan Curve Theorem [7] makes use of a rectangular grid, and Poincaré's description of the Fuchsian groups [8] leans heavily on the observation that the dual graph to the tesselation generated by the fundamental region is a Cayley graph of the group. It is well known by now that such plane embeddings, or in fact any embeddings of graphs on closed oriented surfaces can be described by means of permutations in the following manner. If we consider a graph as a symmetric directed graph, then the orientation of the ambient surface induces a cyclic permutation of the arcs emanating from each vertex of the graph. Let $P$ denote the disjoint product of all these cycles and let $Q$ be the involution that maps each arc to its inverse. For example, if we interpret Figure 1 as a plane graph, then $P=(153)(2 c a)(47 b)(698)$ and $Q=(12)(34)(56)(78)(9 a)(b c)$. Note that the disjoint cycle decomposition of the product $P Q$ is $\left(\begin{array}{lll}1 & 6 & a\end{array}\right)\left(\begin{array}{lll}2 & b & 3\end{array}\right)(485)(7 c$ 9 $)$ and that these cyclic factors are in an obvious one-to-one correspondence with the regions of the embedding. As this correspondence is not merely coincidental, it seems reasonable to expect the symmetric group to play a deep role in the combinatorial topology of the orientable surfaces. Robert Cori $[2,4]$ and others have pointed out that fixed point theorems have interesting analogs for centralizers of certain sets of permutations. The author has discussed the Jordan Curve Theorem in this context in [10] and has also shown in [11] that the characters of the symmetric groups can be brought to bear on the difficult problems involved in the enumeration of combinatorial maps.

In this paper we construct a combinatorial analog of both Euclidean and nonEuclidean tesselations. §I contains a description of fundamental groups and wrappings of ordered sets of permutations. The role of the centralizers of these permutations is explained in §II, which owes much to the work of R. Cori in [2] and elsewhere. This section also includes a discussion of quotient maps and of deck

Received by the editors March 21, 1986.

1980 Mathematics Subject Classification (1985 Revision). Primary 20F05.

This work was supported in part by University of Kansas General Research Allocation \#3188-20-0038. Portions of the article were written while the author was on sabbatical leave at the University of Michigan. The author thanks Professor R. C. Lyndon for his patience and valuable advice. 


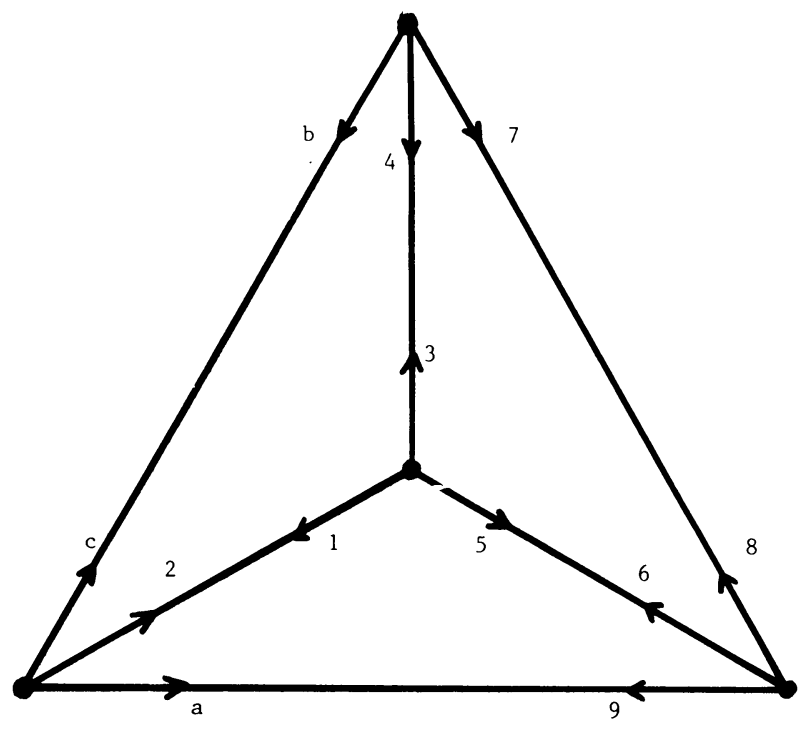

Figure 1

transformations. Canonical maps are defined in §III where it is shown that their groups have the expected presentation in terms of what Poincare called fundamental relations. It is shown in §IV that some well-known groups arise in this manner, including the Fuchsian groups of the first kind (without parabolic generators) and the triangle groups. Two large classes of examples of such wrappings are presented in $\S \mathrm{V}$.

I. Wrappings and fundamental groups. All functions and permutations in this paper are assumed to act on the right, so that their composition is to be read from left to right. The image of an element $a$ under the action of the permutation $P$ will be denoted by $a P$. We define a combinatorial map $M$ to be a triple $(S, b, P)$, where $S$ is a set, $b$ is an element of $S$ that is called the basepoint of $M$, and $P$ is a sequence $\left(P_{1}, P_{2}, \ldots, P_{c}\right)$ of permutations that act transitively on the underlying set $S$.

It should be pointed out that this terminology differs from that found elsewhere in the literature in that it introduces a base point and allows for more than two permutations. The need for the base point will become clear later. The advantage of allowing for more permutations lies, obviously, in its greater generality, especially since this turns out to cause no increase in the complexity of the proofs.

Given two maps $\tilde{M}=(\tilde{S}, \tilde{b}, \tilde{P})$ and $M=(S, b, P)$, where $\tilde{P}=\left(\tilde{P}_{1}, \ldots, \tilde{P}_{c}\right)$ and $P=\left(P_{1}, \ldots, P_{c}\right)$, and given a function $f: \tilde{S} \rightarrow S$, we say that $\tilde{f}$ is a wrapping of $\tilde{M}$ onto $M$ provided that the following conditions hold:

$$
(\tilde{b}) f=b
$$

(I.2) $\quad x \tilde{P}_{i}^{k}=x$ if and only if $(x) f P_{i}^{k}=(x) f$, for all $k$ and all $x \in \tilde{S}$.

The first condition requires no comment. The second says that $\tilde{P}_{i}$ and $P_{i}$ act consistently at corresponding elements of $\tilde{S}$ and $S$. The last condition implies that 
corresponding orbits (cyclic factors) of $P_{i}$ and $\tilde{P}_{i}$ have equal length. Such a wrapping will usually be denoted by $f:(\tilde{S}, \tilde{b}, \tilde{P}) \rightarrow(S, b, P)$ or $f: \tilde{M} \rightarrow M$.

EXAMPLE I.1. Let $\tilde{S}=\{\tilde{1}, \tilde{2}, \tilde{3}, \hat{1}, \hat{2}, \hat{3}\}, S=\{1,2,3\}, \tilde{b}=\tilde{1}, b=1, \tilde{P}_{1}=\left(\begin{array}{lll}\tilde{1} & \tilde{2} & \tilde{3}\end{array}\right)$

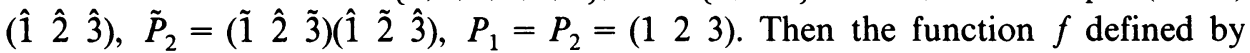
$(\tilde{1}) f=(\hat{1}) f=1,(\tilde{2}) f=(\hat{2}) f=2,(\tilde{3}) f=(\hat{3}) f=3$ exhibits $\tilde{M}=\left(\tilde{S}, \tilde{b},\left(\tilde{P}_{1}, \tilde{P}_{2}\right)\right)$ as a wrapping of $M=\left(S, b,\left(P_{1}, P_{2}\right)\right)$.

EXAMPLE I.2. Let $Z$ denote the set of positive integers and set

$$
\begin{aligned}
& \tilde{S}=\{(i, j, k) / i, j \in Z, k \in\{1,2,3,4\}\}, \\
& \tilde{b}=(0,0,1), \\
& (i, j, k) \tilde{P}_{1}=(i, j, k+1), \quad \text { addition modulo } 4 \\
& (i, j, 1) \tilde{P}_{2}=(i, j+1,3), \\
& (i, j, 2) \tilde{P}_{2}=(i-1, j, 4), \\
& (i, j, 3) \tilde{P}_{2}=(i, j-1,1), \\
& (i, j, 4) \tilde{P}_{2}=(i+1, j, 2), \\
& \tilde{P}=\left(\tilde{P}_{1}, \tilde{P}_{2}\right) .
\end{aligned}
$$

The combinatorial map $\tilde{M}=(\tilde{S}, \tilde{b}, \tilde{P})$ is the one that corresponds to the plane graph whose vertices consist of the integer lattice and whose edges are the unit length lines connecting these vertices. The permutation $\tilde{P}_{1}$ describes the clockwise orientation at each vertex, and $\tilde{P}_{2}$ is the permutation derived from the edges of the embedded graph. Next, set

$$
\begin{aligned}
& S=\{1,2,3,4\}, \\
& b=1, \\
& P=\left(P_{1}, P_{2}\right), \\
& P_{1}=(1234), \quad P_{2}=\left(\begin{array}{ll}
1 & 3
\end{array}\right)(24), \\
& (i, j, k) f=k .
\end{aligned}
$$

Then $f: \tilde{M} \rightarrow M$ is a wrapping, where $M=(S, b, P)$. The combinatorial map $M$ corresponds to the well-known 2-cell embedding of a graph with a single vertex and two loops on the torus.

In $\S \S I$ and II, whenever we refer to maps $M$ and $\tilde{M}$ it will be assumed that $M=(S, b, P), \tilde{M}=(\tilde{S}, \tilde{b}, \tilde{P}), P=\left(P_{1}, P_{2}, \ldots, P_{c}\right)$, and $\tilde{P}=\left(\tilde{P}_{1}, \tilde{P}_{2}, \ldots, \tilde{P}_{c}\right)$. This will actually hold throughout the article, except that in $\S \S I I I$ and IV the constituent permutations of $P$ and $\tilde{P}$ will be more explicitly specified.

Given a map $M$, the orbits of each constitutent permutation $P_{i}$ are referred to as the $P_{i}$-cells. If $f: \tilde{M} \rightarrow M$ is the wrapping described above, then it follows from (I.1) and (I.2) that the restriction of $f$ to each $\tilde{P}_{i}$-cell is a bijection onto the corresponding $P_{i}$-cell. Since (I.1) is still valid when $P_{i}$ is replaced by the product $P_{1} P_{2} \cdots P_{c}$, it is easily seen that $f$ wraps each cell of $\tilde{P}_{1} \tilde{P}_{2} \cdots \tilde{P}_{c}$ around the corresponding cell of $P_{1} P_{2} \cdots P_{c}$. The quotient of the lengths of these corresponding cells is therefore an integer (or is infinite) and is called their wrapping index.

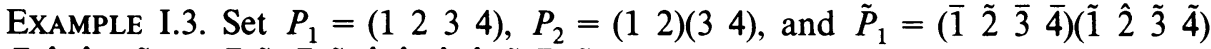

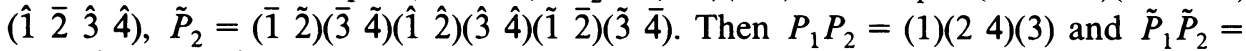
$(\overline{1})(\tilde{1} \hat{1})(\tilde{2} \tilde{4} \overline{2} \hat{4} \hat{2} \overline{4})(\hat{3})(\tilde{3} \overline{3})$. If we set $(\bar{a}) f=(\tilde{a}) f=(\hat{a}) f=a$ for $a \in\{1,2,3,4\}$, 
then $f$ defines a wrapping. The wrapping index of (1) around (1) is 1 , whereas the wrapping index of $(\overline{1} \hat{1})$ and (1) is 2 .

Lemma I.4. Let $f: \tilde{M} \rightarrow M$ be a wrapping, let $P_{i_{1}}, P_{i_{2}}, \ldots, P_{i_{m}}$ be a sequence of permutations from $P$, and let $\tilde{P}_{i_{1}}, \tilde{P}_{i_{2}}, \ldots, \tilde{P}_{i_{m}}$ be the corresponding sequence in $\tilde{P}$. Then,

$$
\left(x \tilde{P}_{i_{1}} \tilde{P}_{i_{2}} \cdots \tilde{P}_{i_{m}}\right) f=(x) f P_{i_{1}} P_{i_{2}} \cdots P_{i_{m}} \quad \text { for all } x \in \tilde{S} .
$$

Proof. This follows from (I.1) by a simple induction on $m$. Q.E.D.

If the wrapping $f:(\tilde{S}, \tilde{b}, \tilde{P}) \rightarrow(S, b, P)$ also satisfies the additional condition:

$$
\tilde{a}\left(\tilde{P}_{1} \tilde{P}_{2} \cdots \tilde{P}_{c}\right)^{k}=\tilde{a} \quad \text { if and only if }(\tilde{a}) f\left(P_{1} P_{2} \cdots P_{c}\right)^{k}=(\tilde{a}) f
$$

for all $k$ and all $\tilde{a} \in \tilde{S}$, then we say that $f$ is a covering. The functions $f$ defined in Examples I.1 and I.2 are in fact such coverings, whereas that of Example I.3 is not a covering. The first part of this article is devoted to the classification of such coverings of a combinatorial map $M$.

For any permutation sequence $P=\left(P_{1}, \ldots, P_{c}\right)$ we define $W(P)$ to be the set of all the words over the symbol set $\left\{P_{i}^{ \pm 1}\right\}_{i=1}^{c}$. We now go on to define an equivalence relation on $W(P)$ that mimics the topological notion of homotopy for paths beginning at a basepoint. For each word $W \in W(P)$ and each element $a \in S$, $a W$ denotes the image of $a$ under the action of $W$ when the latter is regarded as the composition of its constituent permutations. Thus in Example I.3 above, $\tilde{1}_{1} \tilde{P}_{1} \tilde{P}_{2} \tilde{P}_{1}=\overline{2}$ and $\tilde{1} \tilde{P}_{2}^{3} \tilde{P}_{1}=\hat{3}$. The set $H(P)$ consists of all those words $X \in W(P)$ such that $X$ is either a word over $\left\{P_{i}, P_{i}^{-1}\right\}$ for some $i=1,2, \ldots, c$, or else $X$ is a concatenation $X_{1} X_{2} \cdots X_{t}$, where each $X_{j}$ is one of the two words $P_{1} P_{2} \cdots P_{c}, P_{c}^{-1} \cdots P_{2}^{-1} P_{1}^{-1}$. If $W_{1}, W_{2} \in W(P)$ and $X \in H(P)$ are words such that

$$
b W_{1} X=b W_{1},
$$

we then say that $W_{1} X W_{2}$ is elementarily homotopic to $W_{1} W_{2}$. The words $U$, $V \in W(P)$ are said to be homotopic if there is a sequence of words $U=W_{1}$, $W_{2}, \ldots, W_{n}=V$ such that for each $i$ either $W_{i}$ is elementarily homotopic to $W_{i+1}$ or vice versa. In this case we write $U \approx V$. This is clearly an equivalence relation on $W(P)$. Note that if $U$ and $V$ are homotopic words then $b U=b V$.

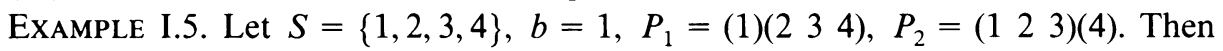
$P_{1} P_{2} \approx P_{2}$ whereas $P_{2} P_{1} P_{2} \approx P_{2} P_{2}$. In fact, for any two words $W_{1}, W_{2} \in W(P)$ we have $W_{1} P_{1}^{3} W_{2} \approx W_{1} P_{2}^{3} W_{2} \approx W_{1}\left(P_{1} P_{2}\right)^{2} W_{2} \approx W_{1} W_{2}$.

The equivalence class (or homotopy class) of the word $W \in W(P)$ is denoted by $[W]$. The set of these homotopy classes is denoted by $\Omega(M)=\{[W] \mid W \in W(P)\}$. In the examples below as well as elsewhere in this article the identity permutation will be denoted by $I$ and the empty word by $\Lambda$.

ExAmple I.6. Let $S=\{1, \ldots, n\}, b=1, P_{1}=(1 \cdots n), P_{2}=(n \cdots 1)$. Then

$$
\Omega(M)=\left\{[\Lambda],\left[P_{1}\right],\left[P_{1}^{2}\right], \ldots,\left[P_{1}^{n-1}\right]\right\}
$$

Example I.7. Let $S=\{1,2,3,4\}, b=1, P_{1}=(12)(34), P_{2}=(13)(24)$. Then

$$
\Omega(M)=\left\{\Lambda,\left[P_{1}\right],\left[P_{2}\right],\left[P_{1} P_{2}\right]\right\} \text {. }
$$


THEOREM I.8. For any map $M$ the binary operation of concatenation defines a group structure on the set $\pi(M)=\{[W] \in \Omega(M) \mid b W=b\}$.

Proof. We first show that the given operation is indeed well defined. Suppose $U_{1} \approx U_{2}$ and $V_{1} \approx V_{2}$. Any chain of elementary homotopies used to establish the equivalence of $U_{1}$ and $U_{2}$ also establishes the equivalence of $U_{1} V_{1}$ and $U_{2} V_{1}$. Moreover, since $b U_{2}=b$, it follows again that every chain of elementary homotopies used to establish the equivalence of $V_{1}$ and $V_{2}$ also establishes the equivalence of $U_{2} V_{1}$ and $U_{2} V_{2}$. Consequently $U_{1} V_{1} \approx U_{2} V_{2}$ and the given binary operation is indeed well defined. The associativity of this operation follows from the associativity of the concatenation of words. Finally, that $\left[W^{-1}\right]$ does indeed provide an inverse to $[W]$ follows from the observation that for each $i$, the deletion of $X=P_{i} P_{i}^{-1}$ or $X=P_{i}^{-1} P_{i}$ from any word $U X V$ constitutes an elementary homotopy. Q.E.D.

The group $\pi(M)$ is called the fundamental group of the map $M$. It is easy to see that the fundamental groups of the maps of Examples I.6 and I.7 are trivial. As the definition of homotopy assumes the choice of a base point, the fundamental group depends on this choice. However, if $b W_{0}=c$ for some $W_{0} \in W(P)$, then it is easily seen that the correspondence $W \leftrightarrow W_{0}^{-1} W W_{0}$ induces an isomorphism between the two fundamental groups based at $b$ and $c$ respectively.

ExAmple I.9. Let $M$ be the map of Example I.2, i.e., $P_{1}=\left(\begin{array}{llll}1 & 2 & 3 & 4\end{array}\right)$ and $P_{2}=$ (1 3)(2 4). Since $P_{2}=P_{1}^{2}$ it follows that a word $W$ in $W(P)$ determines an element of $\pi(M)$ if and only if the sum of the exponents of $P_{1}$ plus twice the sum of the exponents of $P_{2}$ is divisible by 4 . Hence this fundamental group contains $\left[P_{1}^{2} P_{2}\right]=$ $\left[P_{2} P_{1}^{2}\right]^{-1}$ and $\left[P_{1} P_{2} P_{1}\right]=\left[P_{1}^{3} P_{2} P_{1}^{3}\right]^{-1}$. An argument using induction on the length of the word $W$ shows that $P_{1}^{2} P_{2}$ and $P_{1} P_{2} P_{1}$ in fact generate $\pi(M)$. The following reasoning shows that these generators commute:

$$
\begin{aligned}
\left(P_{1}^{2} P_{2}\right)\left(P_{1} P_{2} P_{1}\right)\left(P_{1}^{2} P_{2}\right)^{-1}\left(P_{1} P_{2} P_{1}\right)^{-1} & \approx\left(P_{1}^{2} P_{2}\right)\left(P_{1} P_{2} P_{1}\right)\left(P_{2} P_{1}^{2}\right)\left(P_{1}^{3} P_{2} P_{1}^{3}\right) \\
& \approx P_{1}\left(P_{1} P_{2}\right)^{4} P_{1}^{3} \approx P_{1}^{4} \approx \Lambda .
\end{aligned}
$$

To show that $\pi(M)$ is indeed freely generated by these two elements is more difficult. Instead, we point out that it follows from Propositions II.4 and IV.4 that $\pi(M)$ is the free abelian group on two generators.

We mention in passing that the technique used in [8] for finding a presentation for the edge path group of a simplicial complex can be modified to obtain a presentation for $\pi(M)$. These presentations, however, are rather unwieldly, and so we merely point out that Proposition II.4 and Theorems IV.4 and IV.5 provide us with examples of maps (root maps, to be defined later) with known fundamental groups.

Let $f:(\tilde{S}, \tilde{b}, \tilde{P}) \rightarrow(S, b, P)$ be a covering. The function $f$ induces a one-to-one correspondence between the words of $W(P)$ and those of $W(\tilde{P})$ in which

$$
W=\prod_{j=1}^{l} P_{i_{j}}^{\varepsilon_{j}} \quad \text { is matched with } \quad \tilde{W}=\prod_{j=1}^{l} \tilde{P}_{i_{j}{ }_{j}}^{\varepsilon_{j}} .
$$

We refer to $\tilde{W}$ of (I.4) as the lift of the matching $W$. By Lemma I.4,

$$
(\tilde{b} \tilde{W}) f=b W \quad \text { for all } W \in W(P) .
$$


The correspondence (I.4) is consistent with homotopies; in other words

$$
W_{1} \approx W_{2} \text { in } W(P) \text { if and only if } \tilde{W}_{1} \approx \tilde{W}_{2} \text { in } W(\tilde{P}) \text {. }
$$

To demonstrate this it suffices to work with elementary homotopies. So suppose $W_{1}=U X V$, and $W_{2}=U V$ where $X \in H(P)$ such that $b U X=b U$. The verification of (I.5) reduces to the equivalence of the following two statements

(i): $W_{1}=U X V, W_{2}=U V, b U X=b U$,

(i): $\tilde{W}_{1}=\tilde{U} \tilde{X} \tilde{V}, \tilde{W}_{2}=\tilde{U} \tilde{V}, \tilde{b} \tilde{U} \tilde{X}=\tilde{b} \tilde{U}$.

This last equivalence follows by an application of (I.1), (I.2), and (I.3). In the sequel (I.5) will be referred to as the homotopy lifting property. This property together with (I.4) has the following implication.

THEOREM I.10. Every covering $f: \tilde{M} \rightarrow M$ induces a monomorphism $f_{*}: \pi(\tilde{M}) \rightarrow$ $\pi(M)$ which is defined by

where $\tilde{W}$ is the lift of $W$.

$$
([\tilde{W}]) f_{*}=[W]
$$

Moreover, this induced monomorphism completely determines the covering in the following sense.

TheOREM I.11. Let $M=(S, b, P), \tilde{M}=(\tilde{S}, \tilde{b}, \tilde{P})$, and $\bar{M}=(\bar{S}, \bar{b}, \bar{P})$ be maps and let $f: \tilde{M} \rightarrow M$ and $g: \bar{M} \rightarrow M$ be coverings such that $(\pi(\tilde{M})) f_{*}=(\pi(\bar{M})) g_{*}$. Then there is a bijection $\beta: \tilde{S} \rightarrow \bar{S}$ such that $\beta g=f$ and $(\tilde{b}) \beta=\bar{b}$.

Proof. Two applications of (I.4) establish a one-to-one correspondence between $W(\tilde{M})$ and $W(\bar{M})$ that matches

$$
\tilde{W}=\prod_{j=1}^{l} \tilde{P}_{i_{j}}^{\varepsilon_{i}} \quad \text { with } \quad \bar{W}=\prod_{j=1}^{l} \bar{P}_{i_{j}}^{\varepsilon_{j}} .
$$

In view of (I.5) this correspondence preserves homotopies. For each $x \in \tilde{S}$ let $\tilde{W}_{x}$ be a word in $W(\tilde{P})$ such that $\tilde{b} \tilde{W}_{x}=x$ and define $(x) \beta=\bar{b} \bar{W}_{x}$. We first show that the function $\beta$ is well defined. Let $\tilde{T}_{x}$ be another word in $W(\tilde{P})$ such that $\tilde{b} \tilde{T}_{x}=x$. Then $\tilde{b} \tilde{W}_{x} \tilde{T}_{x}^{-1}=\tilde{b}$ and so $\left[\tilde{W}_{x} \tilde{T}_{x}^{-1}\right] \in \pi(\tilde{M})$. Consequently $\left[W_{x} T_{x}^{-1}\right] \in(\pi(\tilde{M})) f_{*}$ and since it was assumed that $f_{*}$ and $g_{*}$ have the same ranges, it follows that $\left[W_{x} T_{x}^{-1}\right] \in(\pi(\bar{M})) g_{*}$. Therefore, $\bar{b} \bar{W}_{x} \bar{T}_{x}^{-1}=\bar{b}$ and so $\bar{b} \bar{W}_{x}=\bar{b} \bar{T}_{x}$. Thus $\beta$ is indeed well defined. The surjectiveness of $\beta$ follows from the surjectiveness of the correspondence $\tilde{W} \leftrightarrow \bar{W}$. Finally, suppose that $(x) \beta=(y) \beta$, or in other words, $\bar{b} \bar{W}_{x}=$ $\bar{b} \bar{W}_{y}$. It then follows that $\bar{b} \bar{W}_{x} \bar{W}_{y}^{-1}=\bar{b}$ and so, because of the equality of the ranges of $f_{*}$ and $g_{*}$ we have $\tilde{b} \tilde{W}_{x} \tilde{W}_{y}^{-1}=\tilde{b}$. This in turn implies that $x=\tilde{b} \tilde{W}_{x}=\tilde{b} \tilde{W}_{y}=y$ and so $\beta$ is also injective.

The commutativity requirement is verified easily from the above definitions as follows:

$$
(x) \beta g=\left(\tilde{b} \tilde{W}_{x}\right) \beta g=\left(\bar{b} \bar{W}_{x}\right) g=b W_{x}=\left(\tilde{b} \tilde{W}_{x}\right) f=(x) f .
$$

Finally, choosing $W_{\tilde{b}}=\Lambda$ implies that $(\tilde{b}) \beta=\bar{b}$. Q.E.D.

THEOREM I.12. Let $M=(S, b, P)$ be a map. Then for every subgroup $G$ of $\pi(M)$ there is a map $\tilde{M}$ with a covering $f: \tilde{M} \rightarrow M$ such that $(\pi(\tilde{M})) f_{*}=G$. 
Proof. For any two words $W_{1}, W_{2}$ in $W(P)$ define

$$
W_{1} \equiv W_{2} \quad \text { if and only if } b W_{1}=b W_{2} \text { and }\left[W_{1} W_{2}^{-1}\right] \in G .
$$

Since $G$ is a group, this is an equivalence relation on $W(P)$, and the equivalence class of the word $W$ is denoted by $\tilde{W}$. Now set

$$
\begin{aligned}
& \tilde{S}=\{\tilde{W} \mid W \in W(P)\}, \quad \tilde{b}=\tilde{\Lambda}, \\
& (\tilde{W}) \bar{P}_{i}=\widetilde{W P}_{i}, \quad i=1,2, \ldots, c .
\end{aligned}
$$

To see that $\bar{P}_{i}$ is a well-defined function of $\tilde{S}$ into $\tilde{S}$, note that if $\tilde{W}_{1}=\tilde{W}_{2}$ then $\left[\left(W_{1} P_{i}\right)\left(W_{2} P_{i}\right)^{-1}\right]=\left[W_{1} W_{2}^{-1}\right] \in G$, and so $\widetilde{W_{1} P_{i}}=\widetilde{W_{2} P_{i}}$. A reversal of this logic yields the injectiveness of $\bar{P}_{i}$, and its surjectiveness is immediate. Consequently each $\bar{P}_{i}$ is a permutation of $\tilde{S}$. Set $\tilde{P}=\left(\bar{P}_{1}, \bar{P}_{2}, \ldots, \bar{P}_{c}\right)$. The transitivity of $\tilde{P}$ follows from the finiteness of lengths of the words in $W(P)$, and so $\tilde{M}=(\tilde{S}, \tilde{b}, \tilde{P})$ is a map. The function $f: \tilde{M} \rightarrow M$ is defined by $(\tilde{W}) f=b W$. It is clear that $f$ is well defined and $(\tilde{b}) f=b$. For any $\tilde{W} \in \tilde{S}$,

$$
\left((\tilde{W}) \bar{P}_{i}\right) f=\left(W P_{i}\right) f=b \widetilde{W P_{i}}=((\tilde{W}) f) P_{i},
$$

and so condition (I.1) holds. To verify (I.2), suppose first that $(\tilde{W}) \bar{P}_{i}^{k}=\tilde{W}$ for some $\tilde{W}$ in $\tilde{S}$ and some integer $k$. Hence $W P_{i}^{k} \equiv W$ and so $b W P_{i}^{k}=b W$, or $((\tilde{W}) f) P_{i}^{k}=$ $(\tilde{W}) f$. Conversely, if $((\tilde{W}) f) P_{i}^{k}=(\tilde{W}) f$, then $(b W) P_{i}{ }^{k}=b W, W P_{i}^{k} \approx W$, and so $\left[W P_{i}^{k} W^{-1}\right]=[\Lambda] \in G$. It now follows that $\widetilde{W P}_{i}^{k}=\tilde{W}$ and so $(\tilde{W}) \bar{P}_{i}^{k}=\tilde{W}$. Thus the function $f: \tilde{M} \rightarrow M$ defines a wrapping. To see that $f$ is indeed a covering, one need only replace $P_{i}$ with the composition $P_{1} P_{2} \cdots P_{c}$ in the above argument.

It remains to show that $(\pi(\tilde{M})) f_{*}=G$. Let $[W]$, where

$$
W=\prod_{j=1}^{\omega} P_{i_{j}}^{\varepsilon_{j}}
$$

be an arbitrary element of $g$. Then set

$$
\bar{W}=\prod_{j=1}^{\omega} \bar{P}_{i_{j}{ }_{j}}^{\varepsilon_{j}} \in W(\tilde{P}) .
$$

Now $\tilde{b} \bar{W}=\tilde{W}$ because $\tilde{b}=\tilde{\Lambda}$, and $\tilde{W}=\tilde{\Lambda}$ because $[W] \in G$. Consequently $\tilde{b} \bar{W}=\tilde{b}$ and so $\bar{W}$ is an element of $\pi(\tilde{M})$ such that $([\bar{W}]) f_{*}=[W]$. Thus $(\pi(\tilde{M})) f_{*} \supseteq G$. Since the word $\bar{W}$ of (I.6) is in fact an arbitrary element of $\pi(\tilde{M})$, it also follows that $(\pi(\tilde{M})) f_{*} \subseteq G$. Q.E.D.

A covering $f: \tilde{M} \rightarrow M$ is said to be universal if $\pi(\tilde{M})$ is a trivial group. Given such a universal covering. Theorems I.11 and I.12 have the effect of identifying each homotopy class $[W]$ in $\Omega(M)$ with the element $\tilde{b} \tilde{W}$ of $\tilde{S}$. This, of course, identifies the classes in $\pi(M)$ with the elements of $(b) f^{-1}$.

Proposition I.13. Let $\tilde{M}=(\tilde{S}, \tilde{b}, \tilde{P})$ and $\tilde{M}_{1}=\left(\tilde{S}, \tilde{b}_{1}, \tilde{P}\right)$ be two combinatorial maps that differ only in their base point, and let $M=(S, b, P)$ be another combinatorial map. If the function $f: \tilde{S} \rightarrow S$ induces the coverings $f: \tilde{M} \rightarrow M$ and $f: \tilde{M}_{1} \rightarrow M$, then $(\pi(\tilde{M})) f_{*}$ and $\left(\pi\left(\tilde{M}_{1}\right)\right) f_{*}$ are conjugate subgroups of $\pi(M)$. 
Proof. Since $\tilde{P}$ is transitive, there exists a word $\tilde{W} \in W(\tilde{P})$ such that $\tilde{b} \tilde{W}=\tilde{b}_{1}$. If we set $[W]=([\tilde{W}]) f_{*}$ then it is easily seen that $[W] \in \pi(M)$ and that conjugation by $[W]$ does indeed carry $(\pi(\tilde{M})) f_{*}$ onto $\pi\left(\tilde{M}_{1}\right) f_{*}$. Q.E.D.

A covering $f: \tilde{M} \rightarrow M$ is said to be regular if for all elements $x \in(b) f^{-1}$ and for all maps $\tilde{M}_{x}=(\tilde{S}, x, \tilde{P})$, we have $\left(\pi\left(\tilde{M}_{x}\right)\right) f_{*}=(\pi(\tilde{M})) f_{*}$. Given a combinatorial map $M$, a word $W \in W(P)$ is said to be closed at $a \in S$ if $a W=a$; otherwise $W$ is said to be open at $a$.

Proposition I.14. A covering $f: \tilde{M} \rightarrow M$ is regular if and only if for each $\tilde{W} \in W(\tilde{P}), \tilde{W}$ is either closed at all the elements of $(b) f^{-1}$ or open at all of them.

Proof. If $\tilde{M}=(\tilde{S}, \tilde{b}, \tilde{P})$, then set $M_{x}=(\tilde{S}, x, \tilde{P})$ for each $x \in \tilde{S}$. It follows from the homotopy lifting property (I.5) that if $\tilde{W} \in W(\tilde{P})$, then $\tilde{W}$ is closed at $x \in(b) f^{-1}$ if and only if $([\tilde{W}]) f_{*} \in \pi\left(M_{x}\right) f_{*}$. Hence, if the given covering is regular, then since the groups $\left(\pi\left(\tilde{M}_{x}\right)\right) f_{*}$ coincide for all $x \in(b) f^{-1}$, each $\tilde{W} \in$ $W(\tilde{P})$ is either closed at all $x \in(b) f^{-1}$ or at none of them. Conversely, if the covering is not regular, then there exist $x, y \in(b) f^{-1}$ such that $\left(\pi\left(\tilde{M}_{x}\right)\right) f_{*}$ and $\left(\pi\left(\tilde{M}_{y}\right)\right) f_{*}$ are distinct. By symmetry we may assume that there exists a $[W] \in$ $\left(\pi\left(\tilde{M}_{x}\right)\right) f_{*}-\left(\pi\left(\tilde{M}_{y}\right)\right) f_{*}$. The lift $\tilde{W}$ of $W$ is then closed at $x$ and open at $y$. Q.E.D.

II. Automorphisms and quotients. The centralizer of a set $P$ of permutations acting on an underlying set $S$ consists of all the permutations of $S$ that commute with the elements of $P$, and is denoted by $Z(P)$. For a given map $M=(S, b, P)$, the automorphisms of $M$ are the elements of $Z(P)$ where $P$ is regarded as a set. The set of automorphisms of the map $M$ is denoted by $Z(M)$. A deck transformation of the covering $f: \tilde{M} \rightarrow M$ is an element $\delta$ of $Z(\tilde{M})$ such that $\delta f=f$.

EXAmple II.1. The permutation $(\tilde{1} \hat{1})\left(\begin{array}{lll}\tilde{2} & \hat{2}\end{array}\right)\left(\begin{array}{ll}\tilde{3} & \hat{3}\end{array}\right)$ is the only nontrivial deck transformation of the covering in Example I.1.

Proposition II.2. If $\delta$ is a deck transformation of the covering $f: \tilde{M} \rightarrow M$ such that $x \delta=x$ for some $x \in \tilde{S}$, then $\delta$ is the identity permutation.

Proof. Suppose $x \delta=x$ for some $x \in \tilde{S}$. For each $\tilde{P}_{i}$

$$
x \tilde{P}_{i} \delta=x \delta \tilde{P}_{i}=x \tilde{P}_{i} .
$$

Hence each element in each $\tilde{P}_{i}$-orbit of $x$ is also a fixed point of $\delta$. The statement of the proposition now follows from the transitivity of the permutations in $\tilde{P}$. Q.E.D.

It follows from the above proposition that given any $x, y \in \tilde{S}$ there is at most one deck transformation that carries $x$ to $y$. This yields another way of characterizing regular coverings.

THEOREM II.3. The covering $f: \tilde{M} \rightarrow M$ is regular if and only if the deck transformations act transitively on the elements of $(a) f^{-1}$ for each $a \in S$.

Proof. Let $a$ be any element of $S$ and let $W \in W(P)$ be such that $b W=a$. If $\tilde{W}$ is the lift of $W$ as defined by (I.4), then the map $x \rightarrow x \tilde{W}$ is a bijection of $(b) f^{-1}$ and $(a) f^{-1}$ which commutes with the deck transformations. Consequently the deck 
transformations act transitively on $(b) f^{-1}$ if and only if they act transitively on (a) $f^{-1}$ for all $a \in S$. If $f$ is not regular, then there exists a word $\tilde{W}$ in $W(\tilde{P})$ which is closed at $x$ and open at $y$ for some $x, y \in(b) f^{-1}$. However, if $\delta$ is any deck transformation of $f$ such that $x \delta=y$, then the commuting property of $\delta$ implies that $W$ is closed at $x$ if and only if it is closed at $y$. Hence no deck transformation can carry $x$ to $y$. On the other hand, if $f$ is regular, and $x$ is an arbitrary element of (b) $f^{-1}$, then the existence of a deck transformation that carries $\tilde{b}$ to $x$ follows from Theorem I.11. This implies the transitivity of the deck transformations over $(b) f^{-1}$. Q.E.D.

Given a wrapping $f: \tilde{M} \rightarrow M$, elements $c, d \in S$, and a word $W \in W(P)$ such that $c W=d$, let $\tilde{W}$ be the lift of $W$. As was noted in the proof of Theorem II.3, the function $x \rightarrow x \tilde{W}$ defines a bijection between $(c) f^{-1}$ and $(d) f^{-1}$. It follows from the connectedness of $M$ and $\tilde{M}$ that the cardinality of $(a) f^{-1}$ is constant for all $a \in S$. This common cardinality is called the covering index of $f$. It follows from Proposition II.2 that the order of the group of deck transformations of the covering $f: \tilde{M} \rightarrow M$ is bounded above by the covering index. Theorem II.3 implies that equality holds if and only if the covering is regular.

In view of the special role played by universal coverings in the sequel, their deck transformations are discussed in some further detail.

Proposition II.4. The group of deck transformations of the universal covering $f$ : $\tilde{M} \rightarrow M$ is isomorphic to $\pi(M)$.

Proof. We remind the reader that Theorem I.12 identifies the elements of $(b) f^{-1}$ with the elements of $\pi(M)$. For each word $W \in W(P)$ that is closed at $b$, we define a permutation $\lambda_{W}$ of $(b) f^{-1}=\pi(M)$ via the concatenation

$$
\left(\left[W^{\prime}\right]\right) \lambda_{W}=\left[W W^{\prime}\right] \text {. }
$$

Since $W$ acts on the left of $W^{\prime}$ in the above definition, it is clear that $\lambda_{W_{1}}=\lambda_{W_{2}}$ whenever $W_{1}$ and $W_{2}$ are homotopic. Since $W$ is closed at $b, \lambda_{W}$ is well defined on the elements of $\pi(M)$. That $\lambda_{W}$ is in fact a permutation of $(b) f^{-1}=\pi(M)$ follows from the fact that if $V=W^{-1}$ then $\lambda_{V}$ is a two-sided inverse of $\lambda_{W}$. It should be clear at this point that $\lambda_{w}$ is in fact the left regular representation of $\pi(M)$. Since $\lambda_{W}$ acts on the left whereas the $\tilde{P}_{i}$ act on the right, $\lambda_{W}$ commutes with the $\tilde{P}_{i}$. The fact that $b W W^{\prime}=b W^{\prime}$ means that $\lambda_{W} f=f$. Thus each $\lambda_{W}$ is indeed a deck transformation of $f$. If $\lambda_{W}$ is the identity permutation, then $W$ is clearly null homotopic. For any deck transformation $\delta$, let $\tilde{W}$ be a word in $W(\tilde{P})$ such that $\tilde{b} \tilde{W}=\tilde{b} \delta$ (the existence of $\tilde{W}$ is guaranteed by the transitivity of $\tilde{P}$ ). Then $[W]=([\tilde{W}]) f_{*}$ is in $\pi(M)$ and, recalling that $\tilde{b}$ is identified with the empty word in $W(P)$,

$$
(\tilde{b}) \lambda_{W} \delta^{-1}=([\Lambda]) \lambda_{W} \delta^{-1}=[W] \delta^{-1}=[\tilde{b} \tilde{W}] \delta^{-1}=\tilde{b} \delta \delta^{-1}=\tilde{b}
$$

Hence, by Proposition II.2, $\delta=\lambda_{W}$. Since $\lambda_{W_{1} W_{2}}=\lambda_{W_{2}} \lambda_{W_{1}}$, the correspondence $[W] \rightarrow\left(\lambda_{W}\right)^{-1}$ furnishes the required isomorphism. Q.E.D.

We next address the question of which subgroups of the automorphisms can act as the deck transformations of some covering. According to Proposition II.2 nontrivial deck transformations cannot have fixed points. This proposition can be strengthened 
to one that actually does provide a characterization of groups of deck transformations. Given a map $M=(S, b, P)$, if $\zeta$ is an arbitrary automorphism of $M$, then it is well known that $\zeta$ also permutes the orbits of each $P_{i}$ according to the following rule:

$$
\begin{aligned}
\left(\begin{array}{rlll}
a a P_{i} a P_{i}^{2} \cdots & \cdots P_{i}^{k}
\end{array}\right) \zeta & =\left(\begin{array}{llll}
a \zeta a P_{i} \zeta a P_{i}^{2} \zeta & \cdots & a P_{i}^{k} \zeta
\end{array}\right) \\
& =\left(\begin{array}{llll}
a \zeta a \zeta P_{i} a \zeta P_{i}^{2} & \cdots & a \zeta P_{i}^{k}
\end{array}\right) .
\end{aligned}
$$

Here and below, whenever an orbit is written out explicitly, we shall simplify the notation by assuming the orbit to be finite. The interested reader will have no difficulties in making the modifications required to cover the infinite case as well.

Proposition II.5. Let $\Delta$ be the group of deck transformations of the wrapping $f$ : $\tilde{M} \rightarrow M$. Then the action of the nontrivial elements of $\Delta$ on the cells of each $\tilde{P}_{i} \in \tilde{P}$ has no fixed points. If $f$ is a covering, then the action of the nontrivial elements of $\Delta$ on the cells of $\tilde{P}_{1} \tilde{P}_{2} \ldots \tilde{P}_{c}$ also has no fixed points.

Proof. Suppose first that $f: \tilde{M} \rightarrow M$ is a wrapping and let $\delta$ be an automorphism of $f$ such that the induced action of $\delta$ on the cells of some $\tilde{P}_{i} \in \tilde{P}$ has the

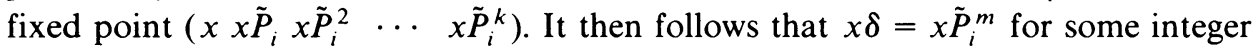
$m$. Then,

$$
(x) f=(x \delta) f=\left(x \tilde{P}_{i}^{m}\right) f .
$$

However, it follows from (I.1) and (I.2) that $f$ maps the elements of each orbit of $\tilde{P}_{i}$ injectively onto the elements of an orbit of the corresponding $P_{i} \in P$. Consequently $x \tilde{P}_{i}^{m}=x$ and so $x \delta=x$. By Proposition II.2, $\delta$ must be the identity.

If $f$ is a covering, then condition (I.3) also holds and it implies, in an analogous manner, the desired result for the action of $\Delta$ on the cells of $\tilde{P}_{1} \tilde{P}_{2} \ldots \tilde{P}_{c}$. Q.E.D.

THEOREM II.6. If $\tilde{M}=(\tilde{S}, \tilde{b}, \tilde{P})$ is a map and $\Delta$ is a subgroup of the automorphism group of $\tilde{M}$ such that the induced action of each nontrivial element of $\Delta$ on the cells of each $\tilde{P}_{i} \in \tilde{P}$ has no fixed points, then there is a wrapping $f: \tilde{M} \rightarrow M$ whose group of deck transformations is $\Delta$. If, in addition, the action of the nontrivial elements of $\Delta$ on the cells of $P_{1} P_{2} \ldots P_{c}$ also has no fixed points, then $f$ is a covering.

Proof. Let $\tilde{M}=(\tilde{S}, \tilde{b}, \tilde{P})$ be a map, and $\Delta$ a subgroup of its automorphism group the induced action of whose elements on the orbits of the permutations in $\tilde{P}$ is fixed point free. Denote by $x \Delta$ the orbit of $x \in \tilde{S}$ under the action of $\Delta$. We set

$$
S=\{x \Delta \mid x \in \tilde{S}\}, \quad b=\tilde{b} \Delta .
$$

For each $\tilde{P}_{i} \in \tilde{P}$ and each $a=x \Delta \in S$, define

$$
a P_{i}=x \tilde{P}_{i} \Delta .
$$

To see that $P_{i}$ is well defined, suppose $a=x \Delta=y \Delta$. Then there is a $\delta \in \Delta$ such that $x \delta=y$ and so

$$
y \tilde{P}_{i} \Delta=x \delta \tilde{P}_{i} \Delta=x \tilde{P}_{i} \delta \Delta=x \tilde{P}_{i} \Delta .
$$

The function $P_{i}$ inherits the invertibility of $\tilde{P}_{i}$ and so it is indeed a permutation. Let $P=\left(P_{1}, P_{2}, \ldots\right)$. 
We now set $M=(S, b, P)$ and define the function $f: \tilde{S} \rightarrow S$ as $(x) f=x \Delta$. Since (x) $f P_{i}=x \Delta P_{i}=x \tilde{P}_{i} \Delta=\left(x \tilde{P}_{i}\right) f$, condition (I.1) is satisfied. Suppose now that $x \Delta P_{i}^{k}=x \Delta$ for some $x \in \tilde{S}, P_{i} \in P$, and some integer $k$. Then

$$
x \tilde{P}_{i}^{k} \Delta=x \Delta
$$

and so there is a $\delta \in \Delta$ such that

$$
x \tilde{P}_{i}^{k}=x \delta .
$$

But this means that the orbit of $\tilde{P}_{i}$ that contains $x$ is fixed by the induced action of $\delta$, and so $\delta$ is the identity,

$$
x \tilde{P}_{i}^{k}=x,
$$

and condition (I.2) is satisfied. Thus, $f$ is a wrapping. The same proof, mutatis mutandis, also shows that condition (I.3) holds when the additional hypothesis regarding the orbits of $\tilde{P}_{1} \tilde{P}_{2} \ldots \tilde{P}_{c}$ is satisfied, so that in that case the wrapping $f$ is in fact a covering.

Since $\Delta$ is by definition a subgroup of $Z(\tilde{M})$ and $x \delta \Delta=x \Delta$ for all $x \in \tilde{S}$ and $\delta \in \Delta$, it is clear that $\Delta$ consists of deck transformations of $f$. Conversely, suppose $\zeta$ is a deck transformation of $f$. Then $(\tilde{b} \zeta) f=(\tilde{b}) f=b$ and so $\tilde{b} \zeta \in(b) f^{-1}=b \Delta$. Hence there exists a $\delta \in \Delta$ such that

$$
\tilde{b} \zeta=\tilde{b} \delta .
$$

As the nonidentity deck transformations have no fixed points in $\tilde{S}$, it follows that $\zeta=\delta$ and so $\Delta$ consists of all the deck transformations of $f$. Q.E.D.

We shall refer to the map $M$ produced in the above proof as a quotient map and denote it by $\tilde{M} / \Delta$.

III. Canonical maps. A map $M=(S, b, P)$ is said to be graphical if $c=2$ and $P_{1}$ is an involution without fixed points. For graphical maps we will dispense with the indices of the permutations in $P$ and simply write $P=(Q, R)$ where $Q=P_{1}$ and $R=P_{2}$. A pair $(\tilde{M}, \Phi)$ is said to be a canonical map if $\tilde{M}=(\tilde{S}, \tilde{b}, \tilde{P})$ is a graphical map with a trivial fundamental group, and $\Phi$ is a subgroup of the automorphisms of $\tilde{M}$ such that

(i) the action of $\Phi$ on the $\tilde{Q}$-cells has no fixed points,

(ii) given any two $\tilde{R}$-cells, there is exactly one element of $\Phi$ that carries one cell onto the other.

This section is devoted to the description of such canonical maps. They are the combinatorial analogs of the canonical tesselations of [5].

It follows from Theorem II.6 that with every canonical map there is associated a wrapping $f: \tilde{M} \rightarrow M$, where $M=\tilde{M} / \Phi$ and where $\Phi$ is the group of deck transformations of $f$. If $M=(S, b, P)$ and $P=(Q, R)$, then it is clear that $Q$ is also an involution without fixed points. Condition (III.2), in conjunction with Proposition II.2, implies that $R$ is a cyclic permutation. If we denote the $\tilde{R}$-cell that contains the base point $\tilde{b}$ of $\tilde{M}$ by $\tilde{R}_{0}$, then we shall follow the convention that the corresponding elements of $\tilde{R}_{0}$ and $R$ will be denoted by $\tilde{a}$ and $a$ respectively. Cells $\tilde{R}_{1}$ and $\tilde{R}_{2}$ of $\tilde{R}$ for which there exists an $x \in \tilde{S}$ such that $x \in \tilde{R}_{1}$ and $x \tilde{Q} \in \tilde{R}_{2}$ are 
said to be adjacent, and we also say that the cells $\tilde{R}_{1}$ and $\tilde{R}_{2}$ split the $\tilde{Q}$-cell ( $x \times \tilde{Q}$ ). Since $\tilde{Q}$ is an involution, adjacency is a symmetric relation. For each $a \in S$ let $\tilde{R}_{\tilde{a} \tilde{Q}}$ be the $\tilde{R}$-cell that contains $\tilde{a} \tilde{Q}$ (thus splitting ( $\tilde{a} \tilde{a} \tilde{Q}$ ) with $\tilde{R}_{0}$ ), and let $\phi_{a}$ be the unique element of $\Phi$ that carries $\tilde{R}_{0}$ to $\tilde{R}_{\tilde{a} \tilde{Q}}$.

The following lemma is the permutations analog of the well-known observation that the dual of a regular tesselation is the Cayley graph of its group.

LEMMA III.1. Given a canonical map $(\tilde{M}, \Phi)$ suppose that for some $x \in \tilde{S}$ and $\phi \in \Phi, x \in\left(\tilde{R}_{0}\right) \phi$. Then $x \tilde{Q} \in\left(\tilde{R}_{0}\right) \phi_{a} \phi$, where $a=(x) f$.

Proof. Let $\tilde{a}=x \phi^{-1} \in \tilde{R}_{0}$. Then, by the definition of $\phi_{a}$,

$$
\tilde{a} \tilde{Q} \in\left(\tilde{R}_{0}\right) \phi_{a} \text {. }
$$

Hence,

$$
x \tilde{Q}=x \phi^{-1} \tilde{Q} \phi=\tilde{a} \tilde{Q} \phi \in\left(\tilde{R}_{0}\right) \phi_{a} \phi . \quad \text { Q.E.D. }
$$

The elements $\phi_{a}$ are called the canonical generators of $\Phi$.

Proposition III.2. Let $(\tilde{M}, \Phi)$ be a canonical map, let $\Gamma$ be the set of its canonical generators, and let $f: \tilde{M} \rightarrow \tilde{M} / \Phi$ be the quotient covering. Then,

(i) $\Gamma$ generates $\Phi$;

(ii) every cell $\left(\begin{array}{ll}x & y\end{array}\right)$ of $\tilde{Q}$ yields a relator $\phi_{a} \phi_{c}$ on $\Gamma$ where $a=(x) f$ and $c=(y) f$;

(iii) every finite cell $\left(x_{1} x_{2} \cdots x_{m}\right)$ of $\tilde{P}=\tilde{R}^{-1} \tilde{Q}$ yields a relator $\phi_{a_{1}} \phi_{a_{2}} \cdots \phi_{a_{m}}$ on $\Gamma$ where $a_{i}=\left(x_{i}\right) f, i=1,2, \ldots, m$.

Proof. (i) Let $\Phi^{\prime}$ be the subgroup of $\Phi$ that is generated by $\Gamma$ and suppose that $\Phi^{\prime} \neq \Phi$. Because of the transitivity of the action of $\tilde{Q}$ and $\tilde{R}$, there is an element $x \in \tilde{S}$ such that

$$
x \in \bigcup\left\{\left(\tilde{R}_{0}\right) \phi \mid \phi \in \Phi^{\prime}\right\} \quad \text { but } \quad x \tilde{Q} \notin \bigcup\left\{\left(\tilde{R}_{0}\right) \phi \mid \phi \in \Phi^{\prime}\right\} .
$$

Let $\bar{\phi}$ be that element of $\Phi^{\prime}$ for which $x \in\left(\tilde{R}_{0}\right) \bar{\phi}$ and let $a=(x) f$. Then $\tilde{a}=x \bar{\phi}^{-1} \in \tilde{R}_{0}$. Let $\tilde{R}_{1}$ and $\tilde{R}_{*}$ be the orbits of $\tilde{R}$ that contain $\tilde{a} \tilde{Q}$ and $x \tilde{Q}$ respectively. By the definition of $\phi_{a}$,

Since $(\tilde{a} \tilde{Q}) \bar{\phi}=(\tilde{a} \bar{\phi}) \tilde{Q}=x \tilde{Q} \in \tilde{R}_{*}$, we have

$$
\left(\tilde{R}_{0}\right) \phi_{a}=\tilde{R}_{1} \text {. }
$$

$$
\left(\tilde{R}_{0}\right) \phi_{a} \bar{\phi}=\left(\tilde{R}_{1}\right) \bar{\phi}=\tilde{R}_{*} .
$$

Thus $\phi_{a} \bar{\phi}$ is an element of $\Phi^{\prime}$ that carries $\tilde{R}_{0}$ to $\tilde{R}_{*}$. Since $x \tilde{Q}$ is an element of $\tilde{R}_{*}$ and was assumed not to be an element of $\bigcup\left\{\left(\tilde{R}_{0}\right) \phi \mid \phi \in \Phi^{\prime}\right\}$ we have arrived at a contradiction of the assumption that $\Phi^{\prime} \neq \Phi$.

(ii) For the given $x, y$, let $\tilde{a}$ be the unique element of $x \Phi \cap \tilde{R}_{0}$ and let $\tilde{c}$ be the unique element of $y \Phi \cap \tilde{R}_{0}$. Let $\phi_{1}$ and $\phi_{2}$ be the unique elements of $\Phi$ such that $(x) \phi_{1}=\tilde{a}$ and $(y) \phi_{2}=\tilde{c}$. Since $\phi_{1}$ and $\phi_{2}$ commute with $\tilde{Q}$, it follows that

$$
\begin{aligned}
& (y) \phi_{1}=(x \tilde{Q}) \phi_{1}=(x) \phi_{1} \tilde{Q}=\tilde{a} \tilde{Q}, \\
& (x) \phi_{2}=(y \tilde{Q}) \phi_{2}=(y) \phi_{2} \tilde{Q}=\tilde{c} \tilde{Q} .
\end{aligned}
$$

Now $(\tilde{a}) \phi_{c} \in\left(\tilde{R}_{0}\right) \phi_{c}$ and

$$
(\tilde{a}) \phi_{1}^{-1} \phi_{2}=(x) \phi_{2}=\tilde{c} \tilde{Q} \in\left(\tilde{R}_{0}\right) \phi_{c},
$$

so by (III.2) $\phi_{c}=\phi_{1}^{-1} \phi_{2}$. A symmetrical argument yields $\phi_{a}=\phi_{2}^{-1} \phi_{1}$, and so $\phi_{a} \phi_{c}=I$. 
(iii) For the given cell there exists an element $\phi \in \Phi$ such that $x_{m} \in\left(\tilde{R}_{0}\right) \phi$. By Lemma III.1 $x_{m} \tilde{Q} \in\left(\tilde{R}_{0}\right) \phi_{a_{m}} \phi$, and consequently

$$
x_{m-1}=x_{m} \tilde{Q} \tilde{R} \in\left(\tilde{R}_{0}\right) \phi_{a_{m}} \phi .
$$

Repeated applications of this reasoning yield

$$
x_{i} \in\left(\tilde{R}_{0}\right) \phi_{a_{i+1}} \phi_{a_{i+2}} \cdots \phi_{a_{m}}, \quad i=1,2, \ldots, m-1
$$

and

$$
x_{m} \in\left(\tilde{R}_{0}\right) \phi_{a_{1}} \phi_{a_{2}} \cdots \phi_{a_{m}} \phi \text {. }
$$

Thus $\phi_{a_{1}} \phi_{a_{2}} \cdots \phi_{a_{m}} \phi=\phi$ and so $\phi_{a_{1}} \phi_{a_{2}} \cdots \phi_{a_{m}}$ is indeed a relator. Q.E.D.

The relators produced by Proposition II.2(ii) are called trivial, whereas those produced by Proposition III.2(iii) are called fundamental. We go on to show that these constitute a complete set of relators for $\Phi$.

Let

$$
W=V_{0} U_{1} V_{1} U_{2} V_{2} \cdots U_{m} V_{m}
$$

where each $U_{i}$ is a word in $\tilde{Q}^{ \pm 1}$ and each $V_{i}$ is a word in $\tilde{R}^{ \pm 1}$. For such $W$ define

$$
(W) \rho=\phi_{a_{m}}^{\varepsilon_{m}} \phi_{a_{m-1}}^{\varepsilon_{m-1}} \cdots \phi_{a_{1}}^{\varepsilon_{1}}
$$

where $i=1,2, \ldots, m, a_{i}=\left(x_{i}\right) f, \quad x_{i}=\tilde{b} V_{0} U_{1} V_{1} \cdots U_{i-1} V_{i-1}$, and $\varepsilon_{i}=1$ or 0 according as $U_{i}$, as a permutation, equals $\tilde{Q}$ or the identity.

Proposition III.3. Let $(\tilde{M}, \Phi)$ be a canonical map. If $W^{\prime}$ and $W$ are homotopic words of $W(\tilde{P})$, then $\left(W^{\prime}\right) \rho$ can be obtained from $(W) \rho$ by the insertion and deletion of trivial and fundamental relators.

Proof. We first show that this is the case when $W$ (defined as in (III.3)) and $W^{\prime}$ are only formally different, in the sense that $W^{\prime}$ is obtained from $W$ by the elimination (or addition) of several $U_{i}$ and $V_{i}$ that are in fact empty. We suppose first that $U_{s}$ is empty for some $s$, and that

$$
W^{\prime}=V_{0} U_{1} V_{1} \cdots U_{s-1}\left(V_{s-1} V_{s}\right) U_{s+1} \cdots U_{m} V_{m} .
$$

Since $U_{s}$ is empty, it acts like the identity and so $\varepsilon_{s}=0$ in $(W) \rho$. With the exception of $x_{s}$, all the $x_{i}$ 's of $W$ are inherited by $W^{\prime}$. Hence,

$$
(W) \rho=\left(W^{\prime}\right) \rho=\phi_{a_{m}}^{\varepsilon_{m}} \cdots \phi_{a_{s+1}}^{\varepsilon_{s+1}} \phi_{a_{s-1}}^{\varepsilon_{s-1}} \cdots \phi_{a_{1}}^{\varepsilon_{1}} .
$$

Next suppose that $V_{s}$ is empty and that

$$
W^{\prime}=V_{0} U_{1} V_{1} \cdots V_{s-1}\left(U_{s} U_{s+1}\right) V_{s+1} \cdots U_{m} V_{m} .
$$

An application of the definition of the function $\rho$ yields

$$
\left(W^{\prime}\right) \rho=\phi_{a_{m}}^{\varepsilon_{m}} \cdots \phi_{a_{s+2}}^{\varepsilon_{s+2}} \phi_{a_{s}}^{\varepsilon_{s}^{\prime}} \phi_{a_{s-1}}^{\varepsilon_{s-1}} \cdots \phi_{a_{1}}^{\varepsilon_{1}} .
$$

Now, if $U_{s}$ acts like the identity, then $\varepsilon_{s}=0$ and $x_{s+1}=\tilde{b} V_{0} \cdots U_{s} V_{s}=\tilde{b} V_{0} \cdots$ $U_{s-1} V_{s-1}=x_{s}$. Moreover, since in this case $U_{s} U_{s+1}$ acts like $U_{s+1}$, we also have $\varepsilon_{s}^{\prime}=\varepsilon_{s+1}$. A substitution of this information into (III.4) and (III.5) shows that $(W) \rho=\left(W^{\prime}\right) \rho$. If, on the other hand, $U_{s}$ acts like $\tilde{Q}$, then $\varepsilon_{s}=1$ and $x_{s+1}=\tilde{b} V_{0}$ $\cdots U_{s} V_{s}=x_{s} U_{s}=x_{s} \tilde{Q}$. Moreover, since in this case $U_{s} U_{s+1}$ and $U_{s+1}$ act differently, $\varepsilon_{s}^{\prime}=1-\varepsilon_{s+1}$. If $\varepsilon_{s+1}=0$, then

$$
(W) \rho=\left(W^{\prime}\right) \rho=\phi_{a_{m}}^{\varepsilon_{m}} \cdots \phi_{a_{s+2}}^{\varepsilon_{s+2}} \phi_{a_{s}} \phi_{a_{s}-1}^{\varepsilon_{s-1}} \cdots \phi_{a_{1}}^{\varepsilon_{1}} .
$$


If $\varepsilon_{s+1}=1$, then

$$
\begin{aligned}
& (W) \rho=\phi_{a_{m}}^{\varepsilon_{m}} \cdots \phi_{a_{s+2}^{\varepsilon_{s+2}} \phi_{a_{s+1}} \phi_{a_{s}} \phi_{a_{s-1}}^{\varepsilon_{s-1}} \cdots \phi_{a_{1}}^{\varepsilon_{1}},}, \\
& \left(W^{\prime}\right) \rho=\phi_{a_{m}}^{\varepsilon_{m}} \cdots \phi_{a_{s+2}}^{\varepsilon_{s+2}} \phi_{a_{s-1}}^{\varepsilon_{s-1}} \cdots \phi_{a_{1}}^{\varepsilon_{1}} .
\end{aligned}
$$

and so $(W) \rho$ and $\left(W^{\prime}\right) \rho$ differ by $\phi_{a_{s+1}} \phi_{a_{s}}$. Since $x_{s+1}=x_{s} \tilde{Q}$ this difference is a trivial relator of $\Phi$.

If $W^{\prime}$ is obtained from $W$ of (III.3) by replacing some $U_{s}$ with $\left(U_{s} \tilde{Q}^{2}\right)$ or some $V_{s}$ with $\left(V_{s} \tilde{R}^{n}\right)$, then $\left(W^{\prime}\right) \rho$ clearly equals $(W) \rho$. Hence elementary homotopies that involve only $\tilde{Q}$ or only $\tilde{R}$ leave the values of $\rho$ unchanged. Suppose finally that $W^{\prime}$ is obtained from $W$ by an elementary homotopy that involves $\tilde{Q} \tilde{R}$. In other words, there exist integers $1 \leqslant s<t \leqslant m$ such that

$$
\begin{aligned}
& U_{s}=U_{s+1}=\cdots=U_{t}=\tilde{Q}, \\
& V_{s}=V_{s+1}=\cdots=V_{t}=\tilde{R}, \\
& \tilde{b} V_{0} U_{1} V_{1} \cdots U_{s-1} V_{s-1}=\tilde{b} V_{0} U_{1} V_{1} \cdots U_{t} V_{t}, \\
& W^{\prime}=V_{0} U_{1} V_{1} \cdots U_{s-1} V_{s-1} U_{t+1} \cdots U_{m} V_{m} .
\end{aligned}
$$

Observe that in this case $\varepsilon_{s}=\varepsilon_{s+1}=\cdots=\varepsilon_{t}=1$ and so

$$
\begin{aligned}
& (W) \rho=\phi_{a_{m}}^{\varepsilon_{m}} \cdots \phi_{a_{t+1}}^{\varepsilon_{t+1}} \phi_{a_{t}} \cdots \phi_{a_{s}} \phi_{a_{s}, 1}^{\varepsilon_{s}} \cdots \phi_{a_{1}}^{\varepsilon_{1}}, \\
& \left(W^{\prime}\right) \rho=\phi_{a_{m}}^{\varepsilon_{m}} \cdots \phi_{a_{t+2}}^{\varepsilon_{t+2}} \phi_{a_{s}}^{\varepsilon_{t+1}} \phi_{a_{s}, 1}^{\varepsilon_{s}} \cdots \phi_{a_{1}}^{\varepsilon_{1}} .
\end{aligned}
$$

However, by III.6,

$$
\begin{aligned}
& x_{s}=x_{t+1}, \\
& x_{i}=x_{i-1} \tilde{Q} \tilde{R}=x_{i-1} \tilde{P}^{-1}, \quad s<i \leqslant t+1 .
\end{aligned}
$$

So $(W) \rho$ and $\left(W^{\prime}\right) \rho$ differ by $\phi_{a} \cdots \phi_{a_{s}}$, where $\left(x_{s} x_{s+1} \cdots x_{t}\right)$ is an orbit of $\tilde{P}^{-1}$ and so $\left(x_{t} x_{t-1} \cdots x_{s}\right)$ is an orbit of $\tilde{P}$. Q.E.D.

The following proposition uses the above function $\rho$ to characterize the relators of the group generated by $\Gamma$.

Proposition III.4. Let $(\tilde{M}, \Phi)$ be a canonical map. If $W$ is a word in $W(\tilde{P})$ that is closed at $\tilde{b}$, then $(W) \rho$ is a relator of $\Phi$. Conversely, each relator of $\Phi$ can be expressed as $(W) \rho$ for some word $W$ in $\tilde{Q}$ and $\tilde{R}$ that is closed at $\tilde{b}$.

Proof. Let the word $W$, closed at $\tilde{b}$, be expressed as in (III.3). Then $(W) \rho$ is given by (III.4). Observe that since $V_{0}$ is a word in $\tilde{R}$ alone it follows that $x_{1} \in \tilde{R}_{0}$. If $\varepsilon_{1}=0$, then both $x_{1} U_{1}$ and $x_{2}=x_{1} U_{1} V_{1}$ are in $\tilde{R}_{0}$; if $\varepsilon_{1}=1$, then by Lemma III.1 both $x_{1} U_{1}$ and $x_{2}$ are in $\left(\tilde{R}_{0}\right) \phi_{a_{1}}$. In either case,

$$
x_{2} \in\left(\tilde{R}_{0}\right) \phi_{a_{1}}^{\varepsilon_{1}} .
$$

By simple induction it follows that

$$
x_{i} \in\left(\tilde{R}_{0}\right) \phi_{a_{i-1}}^{\varepsilon_{i-1}} \cdots \phi_{a_{1}}^{\varepsilon_{1}} \text { for } i=1,2, \ldots, m
$$

and

$$
\tilde{b}=\tilde{b} W=\left(x_{m}\right) U_{m} V_{m} \in\left(\tilde{R}_{0}\right) \phi_{a_{m}}^{\varepsilon_{m}} \cdots \phi_{a_{1}}^{\varepsilon_{1}}=\left(\tilde{R}_{0}\right)(W) \rho
$$


In other words, $(W) \rho$, as an element of $\Phi$, carries $\tilde{R}_{0}$ to an orbit of $\tilde{R}$ that also contains $\tilde{b}$. It follows from (III.2) that $(W) \rho$ must be a relator.

Conversely, let $\phi_{a_{m}} \cdots \phi_{a_{1}}$ be a relator in $\Phi$. For any two elements $c, d \in S$ let $\delta(c, d)$ be the least nonnegative integer $\delta$ such that $c R^{\delta}=d$. Define:

$$
W=\tilde{R}^{\delta\left(b, a_{1}\right)} \tilde{Q} \tilde{R}^{\delta\left(a_{1} Q, a_{2}\right)} \tilde{Q} \cdots \tilde{Q} \tilde{R}^{\delta\left(a_{m-1} Q, a_{m}\right)} \tilde{Q} \tilde{R}^{\delta\left(a_{m} Q, b\right)} .
$$

Set

$$
\left\{\begin{array}{l}
x_{1}=\tilde{b} \tilde{R}^{\delta\left(b, a_{1}\right)} \\
x_{i}=x_{i-1} \tilde{Q} \tilde{R}^{\delta\left(a_{i-1} Q, a_{i}\right)}, \quad i=2,3, \ldots, m .
\end{array}\right.
$$

Then $x_{1} \in \tilde{R}_{0}$ and

$$
\left(x_{1}\right) f=\left(\tilde{b} \tilde{R}^{\delta\left(b, a_{1}\right)}\right) f=b R^{\delta\left(b, a_{1}\right)}=a_{1} .
$$

A simple induction using Lemmas I.4 and III.1 yields

$$
\left\{\begin{array}{l}
\left(x_{i}\right) f=a_{i}, \\
x_{i} \in\left(\tilde{R}_{0}\right) \phi_{a_{i-1}} \cdots \phi_{a_{1}},
\end{array} \quad \text { for } i=1,2, \ldots, m,\right.
$$

and

$$
\left\{\begin{array}{l}
(\tilde{b} W) f=\left(x_{m} \tilde{Q} \tilde{R}^{\delta\left(a_{m} Q . b\right)}\right) f=a_{m} Q R^{\delta\left(a_{m} Q . b\right)}=b, \\
\tilde{b} W \in\left(\tilde{R}_{0}\right) \phi_{a_{m}} \cdots \phi_{a_{1}}=\tilde{R}_{0} .
\end{array}\right.
$$

These last two conditions imply that $\tilde{b} W=\tilde{b}$, and so $W$ is closed at $\tilde{b}$. It is easily verified that $(W) \rho=\phi_{a_{m}} \cdots \phi_{a_{1}}$ and the details are omitted. Q.E.D.

Since the canonical map $(\tilde{M}, \Phi)$ has a trivial fundamental group, the contents of Propositions III.2, III.3, and III:4 can be summarized in the following statement.

THEOREM III.5. Every canonical map $(\tilde{M}, \Phi)$ yields a presentation of $\Phi$ whose generators are the canonical generators and whose relators are either trivial or fundamental.

We shall refer to the above presentation as the natural presentation of $\Phi$.

IV. $F$-presentations. In a group generated by the elements $\left\{g_{i}\right\}_{i=1}^{s}$, a set of words is said to be orientably strictly quadratic if each of the symbols $\left\{g_{i}, g_{i}^{-1}\right\}, i=$ $1,2, \ldots, s$, occurs exactly once in that set. Let $G=\left\langle g_{1}, \ldots, g_{s} \mid G_{1}^{m_{1}}, \ldots, G_{r}^{m_{r}}\right\rangle$ be a group presentation wherein $G_{1}, G_{2}, \ldots, G_{r}$ form an orientably quadratic set of words. Let $S$ denote the symbol set $\left\{g_{i}^{ \pm 1}\right)_{i=1}$ and let $Q$ be the involution $\left(g_{1}\right.$ $\left.g_{1}^{-1}\right) \cdots\left(g_{s} g_{s}^{-1}\right)$. If $G_{j}=g_{i_{1}} \cdots g_{i_{k_{j}}}$ for $j=1, \ldots, r$, let $P$ be the permutation of $S$ whose cyclic factorization is $P=P_{1} \cdots P_{r}$ where $P_{j}=\left(\begin{array}{llll}g_{i_{1}} & g_{i_{2}} & \cdots & g_{i_{k_{j}}}\end{array}\right)$. Finally, set $R=Q P^{-1}$. If the permutations $P$ and $Q$ act transitively on the set $S$, we then have a graphical $M(G)=\left(S, g_{1},(Q, R)\right)$ which we call the root map of $G$. It is clear that the action of $P$ and $Q$ is transitive if and only if the group presented by $G$ is not the free product of two nontrivial subgroups. The above presentation $G$ is said to be an orientable F-presentation if the product $R=Q P^{-1}$ has a single cell. It is 
easily seen that the group defined by an $F$-presentation is an $F$-group in the sense of [3]. It follows from Propositions III.2, III.3, and III.4 that the natural presentations of the groups of the canonical maps are in fact $F$-presentations.

We now go on to the construction of canonical maps whose groups are to have a prespecified $F$-presentation. This will be accomplished by constructing a suitable wrapping $\bar{f}: \bar{M} \rightarrow M$, a universal covering $\tilde{f}: \tilde{M} \rightarrow \bar{M}$, and then examining the automorphisms of the composition $\tilde{f f}: \tilde{M} \rightarrow M$.

A wrapping $\bar{f}: \bar{M} \rightarrow M$, is said to be uniform if for each $a \in S$, all the elements

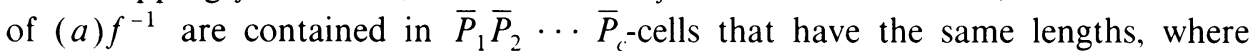
$\bar{M}=(\bar{S}, \bar{b}, \bar{P})$ and $\bar{P}=\left(\bar{P}_{1}, \ldots, \bar{P}_{c}\right)$.

THEOREM IV.1. Let $\tilde{f}: \tilde{M} \rightarrow \bar{M}$ be a universal covering, let $\bar{f}: \bar{M} \rightarrow M$ be a uniform wrapping of the graphical map $M$, and let $f$ be their composition $\tilde{f} \bar{f}$. Then for every $x \in(b) f^{-1}$ there is an automorphism $\zeta \in Z(\tilde{M})$ such that $\zeta f=f,(\tilde{b}) \zeta=x$, and the induced action of $\zeta$ on the $\bar{Q}$-cells has no fixed points.

Proof. Let $\bar{M}=(\bar{S}, \bar{b}, \bar{P})$. Since $M$ is graphical $\bar{P}$ also consists of only two permutations $\bar{Q}$ and $\bar{R}$, of which $\bar{Q}$ is an involution without fixed points. Set $\bar{P}=\bar{R}^{-1} \bar{Q}=(\bar{Q} \bar{R})^{-1}$. We first show that for each $\bar{W}_{0} \in W(\bar{P})$ for which $\left(\bar{b} \bar{W}_{0}\right) \bar{f}=$ $(\bar{b}) \bar{f}$, the function defined by

$$
[\bar{W}] \rightarrow\left[\bar{W}_{0} \bar{W}\right]
$$

is a well-defined permutation of the homotopy classes of $W(\bar{P})$. To do this we must show that if $\bar{W}_{1} \approx \bar{W}_{2}$ then $\bar{W}_{0} \bar{W}_{1} \approx \bar{W}_{0} \bar{W}_{2}$. It may clearly be assumed that $\bar{W}_{2}=$ $\bar{W}_{1} X^{k}$, where $X \in\left\{(\bar{Q} \bar{R})^{ \pm 1}, \bar{Q}^{ \pm 1}, \bar{R} \pm 1\right\}$, and $\bar{b} \bar{W}_{1} X^{k}=\bar{b} \bar{W}_{1}$. Hence,

$$
\bar{b} \bar{W}_{0} \bar{W}_{2}=\bar{b} \bar{W}_{0} \bar{W}_{1} X^{k} .
$$

However, by the given condition on $\bar{W}_{0},\left(\bar{b} \bar{W}_{0}\right) \bar{f}=(\bar{b}) \bar{f}$, so by Lemma I.4 $\left(\bar{b}_{W_{0}} \bar{W}_{1}\right) \bar{f}$ $=\left(\bar{b} \bar{W}_{1}\right) \bar{f}$. The uniformity of $\bar{f}$ in turn implies that $\bar{b} \bar{W}_{0} \bar{W}_{1}$ and $\bar{b} \bar{W}_{1}$ are contained in $\bar{P}$-cells that have the same length. Since the map $M$ is graphical and $\bar{M}$ is a wrapping of $M$, all the $\bar{Q}$-cells have the same length (two) and all the $\bar{R}$-cells have the same length (the length of $R$ ). Consequently for every integer $m$,

$$
\bar{b} \bar{W}_{0} \bar{W}_{1} X^{m}=\bar{b} \bar{W}_{0} \bar{W}_{1} \quad \text { if and only if } \bar{b} \bar{W}_{1} X^{m}=\bar{b} \bar{W}_{1} \text {. }
$$

Since we already know that $\bar{b} \bar{W}_{1} X^{k}=\bar{b} \bar{W}_{1}$, it follows that $\bar{b} \bar{W}_{0} \bar{W}_{1} X^{k}=\bar{b} \bar{W}_{0} \bar{W}_{1}$ and so $\bar{W}_{0} \bar{W}_{1} \approx \bar{W}_{0} \bar{W}_{1} X^{k}=\bar{W}_{0} \bar{W}_{2}$. This establishes the well-definedness of the above function. It is clear that for the above $\bar{W}_{0}$, the word $\bar{W}_{0}^{-1}$ also satisfies the condition $\left(\bar{b} \bar{W}_{0}^{-1}\right) \bar{f}=(\bar{b}) \bar{f}$ and that the function it induces inverts that induced by $\bar{W}_{0}$. Thus these associations are indeed permutations of the homotopy classes of $W(\bar{P})$, and so also of $\tilde{S}$.

Suppose now that $x$ is an arbitrary element of $(b) f^{-1}$ and that $\tilde{b} \tilde{W}_{x}=x$ for $\tilde{W}_{x} \in W(\tilde{P})$. Set $\left[\bar{W}_{x}\right]=\left(\left[\tilde{W}_{x}\right]\right) \tilde{f}_{*}$, so that $\bar{W}_{x} \in W(\bar{P})$. Then

$$
(\bar{b}) \bar{f}=(\tilde{b}) f=b=(x) \tilde{f} \bar{f}=\left(\tilde{b} \tilde{W}_{x}\right) \tilde{f} \bar{f}=\left(\bar{b} \bar{W}_{x}\right) \bar{f},
$$

and so, by the first part of the proof, the map $([\bar{W}]) \zeta=\left[\bar{W}_{x} \bar{W}\right]$ is a permutation of the homotopy classes of $W(\bar{P})$. Since $\tilde{b}$ is identified with the empty word in $W(\bar{P})$ it is clear that $(\tilde{b}) \zeta=x$ and so, since $\zeta$ clearly commutes with $\bar{Q}$ and $\tilde{R}$, it remains to 
show that $\zeta f=f$. But for any $\bar{W} \in W(\bar{P})$,

$$
([\bar{W}]) \zeta f=\left(\left[\bar{W}_{x} \bar{W}\right]\right) f=\left(\left[\overline{W_{x}} \bar{W}\right]\right) \tilde{f f}=\left(\bar{b} \bar{W}_{x} \bar{W}\right) f
$$

and, since $\left(\bar{b} \bar{W}_{x}\right) \bar{f}=(\bar{b}) \bar{f}=b$, it follows from Lemma I.4 that

$$
\left(\bar{b} \bar{W}_{x} \bar{W}\right) \bar{f}=(\bar{b} \bar{W}) \bar{f}=([\bar{W}]) \tilde{f f}=([\bar{W}]) f,
$$

and so $\zeta f=f$.

We already know from Proposition II. 2 that, unless $\zeta$ is the identity, the action of $\zeta$ on $\tilde{S}$ has no fixed points. If $\zeta$ fixes a $\tilde{Q}$-cell there must exist a word $\tilde{W} \in W(\tilde{P})$ such that $(\tilde{b} \tilde{W}) \tilde{Q}=(\tilde{b} \tilde{W}) \zeta$. This, in turn, yields

$$
((\tilde{b} \tilde{W}) f) Q=(\tilde{b} \tilde{W} \tilde{Q}) f=(\tilde{b} \tilde{W} \zeta) f=(\tilde{b} \tilde{W}) \zeta f=(\tilde{b} \tilde{W}) f .
$$

Thus $(\tilde{b} \tilde{W}) f$ is a fixed point of $Q$, which is impossible. Q.E.D.

THEOREM IV.2. Let $G=\left\langle g_{1}, \ldots, g_{s} \mid G_{1}^{m_{1}}, \ldots, G_{r}^{m_{r}}\right\rangle$ be an F-presentation, and let $M=\left(S, g_{1},(Q, R)\right)$ be its root map. Suppose there exists a uniform wrapping $\bar{f}$ : $\bar{M} \rightarrow M$ such that the wrapping index around the cell $P_{j}^{-1}$ of $P^{-1}=Q R$ is $m_{j}$, $j=1, \ldots, r$. Then there exists a canonical map $(\tilde{M}, \Phi)$ such that $G$ is the natural presentation of $\Phi$.

Proof. Let $f: \tilde{M} \rightarrow \bar{M}$, where $\tilde{M}=(\tilde{S}, \tilde{b},(\tilde{Q}, \tilde{R}))$, be the universal covering map, and set $f=\tilde{f} \tilde{f}$. Let $\Phi=\{\phi \in Z(\tilde{M}) \mid \phi f=f\}$. Every $\tilde{R}$-cell is a lift of the unique $R$-cell and so contains exactly one element of $\left(g_{1}\right) f^{-1}$. By Theorem IV.1, for any $x \in\left(g_{1}\right) f^{-1}$, there is a corresponding $\phi \in \Phi$ such that $(\tilde{b}) \phi=x$. Since each $\tilde{R}$-cell contains exactly one element of $\left(g_{1}\right) f^{-1}$ it follows from Proposition II. 2 that condition (III.2) is satisfied. Condition (III.1) is given directly by the statement of Theorem IV.1. Hence the pair $(\tilde{M}, \Phi)$ is a canonical map, and the natural presentation of $\Phi$, by Theorem II.5, is indeed $G$. Q.E.D.

We now go on to construct some well-known $F$-presentations by specifying the corresponding uniform wrappings.

LEMmA IV.3. Let $M=(S, b,(Q, R))$ be a graphical map in which the permutation $R$ has a single cell. Let $P_{1} P_{2} \cdots P_{p}$ be the disjoint cycle decomposition of $P=R^{-1} Q$ with each cell $P_{i}$ having $p_{i}$ elements. Let $n^{\prime}, m_{1}, \ldots, m_{p}$ be positive integers, and let $n=|S|$. For each $a \in S$ let $\kappa_{a}$ and $\rho_{a}$ be permutations of $1,2, \ldots, n^{\prime}$ such that

$$
\kappa_{a} \kappa_{a Q}=I=\rho_{a} \rho_{a R} \rho_{a R^{2}} \cdots \rho_{a R^{n-1}}
$$

and such that all the orbits of

$$
\kappa_{a} \rho_{a Q} \kappa_{a P^{-1}} \rho_{a P^{-1} Q} \cdots \kappa_{a P^{-m_{i}+1}} \rho_{a P^{-m_{i}+1} Q}
$$

have length $p_{i} m_{i}$ whenever $a \in P_{i}$. Then there is a uniform wrapping $f: \bar{M} \rightarrow M$ such that each wrapping index around each $P_{i}$ is $m_{i}$.

Proof. Let $\bar{S}=S \times\left\{1,2, \ldots, n^{\prime}\right\}$ and define permutations $\bar{Q}$ and $\bar{R}$ of $\bar{S}$ as

$$
(a, j) \bar{Q}=\left(a Q, j \kappa_{a}\right), \quad(a, j) \bar{R}=\left(a R, j \rho_{a}\right) .
$$

If we set $\bar{b}=(b, 1)$ then, in view of (IV.1), the projection $(a, j) f=a$ clearly defines a wrapping $f: \bar{M} \rightarrow M$ where $\bar{M}=(\bar{S}, \bar{b},(\bar{Q}, \bar{R}))$. Moreover, condition (IV.2) guarantees that this wrapping is indeed uniform. Q.E.D. 
The following two theorems show that the well-known presentation below occurs as a natural presentation of a canonical map.

$$
\begin{aligned}
G=\left\langle x_{1}, \ldots, x_{p}, y_{1}, \ldots, y_{2 n}\right| x_{1}^{m_{1}}, \ldots, & x_{p}^{m_{p}}, \\
& \left.x_{1} \cdots x_{p} y_{1} y_{2} y_{1}^{-1} y_{2}^{-1} \cdots y_{2 n-1} y_{2 n} y_{2 n-1}^{-1} y_{2 n}^{-1}\right\rangle
\end{aligned}
$$

This presentation includes the (orientable) Fuchsian groups, excluding those that are free products of cyclic groups, and also includes the triangle groups.

THEOREM IV.4. Let $e$ be a nonnegative integer, $\gamma$ a positive integer, and for each $i=1,2, \ldots$, e let $m_{i}$ be a positive integer. Then the $F$-presentation

$$
\begin{array}{r}
\left\langle g_{1}, g_{2}, \ldots, g_{2 e+4 \gamma}\right| g_{1}^{m_{1}}, g_{2}^{m_{2}}, \ldots, g_{e}^{m_{e}}, g_{e+1} g_{e+2} \cdots g_{2 e+4 \gamma}, g_{1} g_{e+1}, g_{2} g_{e+2}, \ldots, \\
\left.g_{e} g_{2 e}, g_{2 e+1} g_{2 e+3}, g_{2 e+2} g_{2 e+4}, \ldots, g_{2 e+4 \gamma-2} g_{2 e+4 \gamma}\right\rangle
\end{array}
$$

is the natural presentation of the group of some canonical map.

Proof. If we regard the length 2 relators as merely defining inverses, then the permutations of the root map of the given presentation are

$$
\begin{aligned}
& P=\left(g_{1}\right)\left(g_{2}\right) \cdots\left(g_{e}\right)\left(g_{e+1} g_{e+2} \cdots g_{2 e+4 \gamma}\right), \\
& Q=\left(g_{1} g_{e+1}\right) \cdots\left(g_{e} g_{2 e}\right)\left(g_{2 e+1} g_{2 e+3}\right) \cdots\left(g_{2 e+4 \gamma-2} g_{2 e+4 \gamma}\right), \\
& R=Q P^{-1}
\end{aligned}
$$

and it is easily verified that $R$ is a cyclic permutation. Let $\Sigma_{n^{\prime}}$ denote the symmetric group on $\left\{1,2, \ldots, n^{\prime}\right\}$.

If $e=0$, set $n^{\prime}=1$ and let $\kappa_{g_{1}}=\rho_{g_{i}}=(1)$ for all $i=1, \ldots, 2 e+4 \gamma$.

If $e>0$ let $n^{\prime}=2$ L.C.M. $\left\{m_{1}, m_{2}, \ldots, m_{e}\right\}$ and set

$$
\rho_{g_{1}}=I \in \Sigma_{n^{\prime}}, \quad i=1, \ldots, 2 e+4 \gamma .
$$

Let $\dot{\kappa}_{g_{1}}, i=1, \ldots, e$, be any regular permutation (all cells having the same length) of order $m_{i}$. Since $n^{\prime} / m_{i}$ is an even integer, each $\kappa_{g_{i}}$ is an even permutation and so is their product $\kappa_{g_{1}} \cdots \kappa_{g_{e}}$. Consequently [12] there exist two cyclic permutations $C, D$ in $\Sigma_{n^{\prime}}$ such that $C D=\kappa_{g_{1}} \cdots \kappa_{g_{e}}$. Since the permutations $C^{-1}$ and $D$ have the same cycle structure, they are conjugate, and so there exists another permutation $A$ in $\Sigma_{n^{\prime}}$ such that $D=A C^{-1} A^{-1}$. Now set

$$
\begin{aligned}
& \kappa_{g_{2 e+1}}=\kappa_{g_{2 e+3}}^{-1}=C, \quad \kappa_{g_{2 e+2}}=\kappa_{g_{2 e+4}}^{-1}=A \\
& \kappa_{g_{l}}=I \in \Sigma_{n^{\prime}}, \quad i=2 e+5, \ldots, 2 e+4 \gamma .
\end{aligned}
$$

It is clear that in all cases equations (IV.1) and (IV.2) of Lemma IV.3 are satisfied and so we are done. Q.E.D.

THEOREM IV.5. Let $e$ be an integer greater than 2 , and let $m_{1}, m_{2}, \ldots, m_{e}$ be arbitrary integers greater than 1 . Then the F-presentation

$$
\left\langle g_{1}, g_{2}, \ldots, g_{2 e} \mid g_{1}^{m_{1}}, \ldots, g_{e}^{m_{e}}, g_{e+1} \cdots g_{2 e}, g_{1} g_{e+1}, \ldots, g_{e} g_{2 e}\right\rangle
$$

is the natural presentation of the group of some canonical map. 
Proof. Here

$$
\begin{aligned}
& P=\left(g_{1}\right) \cdots\left(g_{e}\right)\left(g_{e+1} g_{e+2} \cdots g_{2 e}\right), \\
& Q=\left(g_{1} g_{e+1}\right) \cdots\left(g_{e} g_{2 e}\right), \quad R=Q P^{-1},
\end{aligned}
$$

and it is easily verified that $R$ is a cyclic permutation.

By a theorem of G. A. Miller, $[\mathbf{1}, \mathbf{6}]$, given any three integers $l, m, n$, all greater than 1 , there exists an integer $s$ and permutations $L, M, N \in \Sigma_{s}$, of orders $l, m, n$, respectively, such that $L M=N$. By a simple induction argument it follows that there exists an integer $n^{\prime}$ and permutations $K_{g_{i}}$ of order $m_{i}, i=1, \ldots, e$, such that the composition $K_{g_{1}} K_{g_{2}} \cdots K_{g_{e}}$ has order $e$. If we let $\kappa_{g_{i}}$ denote the representative of $K_{g_{i}}$ in the right regular representation of $\Sigma_{s}$, then each of the $\kappa_{g_{i}}$ is regular of order $m_{i}$ and the composition $\kappa_{g_{1}} \cdots \kappa_{g_{e}}$ is regular of order $e$. If we now set

$$
\begin{aligned}
& \kappa_{g_{e+i}}=\kappa_{g_{i}}^{-1}, \quad i=1,2, \ldots, e, \\
& \rho_{g_{i}}=I, \quad i=1,2, \ldots, 2 e,
\end{aligned}
$$

then the conditions of Lemma IV.3 are clearly satisfied and the proof is complete. Q.E.D.

Of particular interest is the case $e=0$ in Theorem IV.4. In that case the group of the presentation is the fundamental group of the closed orientable surface of genus $\gamma$, and the associated permutations are

$$
\begin{aligned}
& P=\left(\begin{array}{lll}
g_{1} g_{2} \cdots & g_{4 \gamma}
\end{array}\right), \\
& Q=\left(\begin{array}{ll}
g_{1} & g_{3}
\end{array}\right)\left(\begin{array}{ll}
g_{2} & g_{4}
\end{array}\right) \cdots\left(g_{4 \gamma-2} g_{4 \gamma}\right), \\
& R=Q P^{-1} .
\end{aligned}
$$

For $\gamma=1$ this specializes to Example I.2, thus proving that the group described in that example is indeed free abelian on two generators.

\section{Examples.}

Topological covering projections. Let $p:(\tilde{X}, \tilde{x}) \rightarrow(X, x)$ be a covering of closed orientable surfaces and let $G$ be a graph that is 2-cell [13] embedded on $X$ so that $x$ is a vertex of $G$. If we associate with $G$ and $p^{-1}(G)$ pairs of permutations and combinatorial maps as described in the introduction, then $p$ induces a combinatorial covering between these combinatorial maps. If $p$ is only a branched covering, and each region of $G$ contains at most one branch point, and $G$ contains no branch points, then $p$ induces a wrapping between the associated combinatorial maps.

Group homomorphisms. Let $h: \tilde{\Gamma} \rightarrow \Gamma$ be a group homomorphisms, and let $\left\{\gamma_{i}\right\}_{i=1}^{c}$ be elements of $\tilde{\Gamma}$ such that

$$
\operatorname{Order}_{\tilde{\Gamma}}\left(\gamma_{i}\right)=\operatorname{Order}_{\Gamma}\left(h\left(\gamma_{i}\right)\right) \text {. }
$$

If $\tilde{S}$ is the subgroup of $\tilde{\Gamma}$ generated by $\left\{\gamma_{i}\right\}_{i=1}^{c}$ and $S=h(\tilde{S})$, let $\tilde{P}_{i}$ be the permutation of $\tilde{S}$ that right multiplication by $\gamma_{i}$ defines, and let $P_{i}$ be the permutation of $S$ that right multiplication by $h\left(\gamma_{i}\right)$ defines. If $\tilde{e}$ and $e$ are the respective identity elements of $\tilde{\Gamma}$ and $\Gamma$, and if $\tilde{P}=\left(\tilde{P}_{1}, \tilde{P}_{2}, \ldots, \tilde{P}_{c}\right)$ and $P=$ $\left(P_{1}, P_{2}, \ldots, P_{c}\right)$, then the restriction of $h$ to $\tilde{S}$ defines a wrapping

$$
f:(\tilde{S}, \tilde{e}, \tilde{P}) \rightarrow(S, e, P) .
$$


If

$$
\operatorname{Order}_{\tilde{\Gamma}}\left(\gamma_{1} \gamma_{2} \cdots \gamma_{c}\right)=\operatorname{Order}_{\Gamma}\left(h\left(\gamma_{1} \gamma_{2} \cdots \gamma_{c}\right)\right)
$$

then $f$ is a covering.

\section{REFERENCES}

1. J. L. Brenner and R. C. Lyndon, Orbits of the product of two permutations, European J. Combin. 4 (1983), 279-293.

2. R. Cori, A. Machi, L. G. Penaud and B. Vauquelin, On the automorphism group of a planar hypermap, European J. Combin. 2 (1981), 331-334.

3. R. C. Lyndon and P. E. Schupp, Combinatorial group theory, Ergeb. Math. Grenzgeb. (3) 89 (1977).

4. A. Machi, Homology of hypermaps, J. London Math. Soc. (2) 31 (1985), 10-16.

5. W. Magnus, Noneuclidean tesselations and their groups, Academic Press, New York, 1974.

6. G. A. Miller, On the product of two substitutions, Amer. J. Math. 22 (1900), 185-190.

7. M. H. A. Newman, Elements of the topology of plane sets of points, Cambridge University Press, New York, 1961.

8. H. Poincaré, Théorie des groupes Fuchsiennes, Acta Math. 1 (1982), 1-62.

9. H. Seifert and W. Threlfall, A textbook of topology, Academic Press, New York, 1980.

10. S. Stahl, A combinatorial analog of the Jordan Curve Theorem, J. Combin. Theory (B) 35 (1983), $28-38$.

11. The average genus of classes of graph embeddings, Congr. Numer. 40 (1983), 375-388.

12. D. W. Walkup, How many ways can a permutation be factored into two n-cycles? Discrete Math. 28 (1979), 315-319.

13. A. T. White, Graphs, groups and surfaces, North-Holland Mathematical Studies 8, North-Holland, Amsterdam, 1984.

Department of Mathematics, University of Kansas, Lawrence, Kansas 66045 